\title{
Transient cerebral ischemia induces albumin expression in microglia only in the CA1 region of the gerbil hippocampus
}

\author{
JOON HA PARK ${ }^{1 *}$, JIN-A PARK $^{2 *}$, JI HYEON AHN ${ }^{1}$, YANG HEE KIM ${ }^{3}$, IL JUN KANG ${ }^{4}$, \\ MOO-HO WON ${ }^{5}$ and CHOONG-HYUN LEE ${ }^{2}$ \\ ${ }^{1}$ Department of Biomedical Science, Research Institute of Bioscience and Biotechnology, Chuncheon, Gangwon 24252; \\ ${ }^{2}$ Department of Pharmacy, College of Pharmacy, Dankook University, Cheonan, South Chungcheong 31116; \\ ${ }^{3}$ Department of Surgery, School of Medicine, Kangwon National University, Chuncheon, Gangwon 24341; \\ ${ }^{4}$ Department of Food Science and Nutrition, Hallym University, Chuncheon, Gangwon 24252; ${ }^{5}$ Department of \\ Neurobiology, School of Medicine, Kangwon National University, Chuncheon, Gangwon 24341, Republic of Korea
}

Received April 21, 2016; Accepted February 9, 2017

DOI: $10.3892 / \mathrm{mmr} .2017 .6671$

\begin{abstract}
Albumin, the most abundant plasma protein, is known to exhibit a neuroprotective effect in animal models of focal and global cerebral ischemia. In the present study, the expression and immunoreactivity of albumin was examined in the hippocampus following 5 min of transient cerebral ischemia in gerbils. Albumin immunoreactivity was observed in microglia of the CA1 hippocampal region 2 days post-ischemic insult, and it was significantly increased at 4 days following ischemia-reperfusion. In addition, at 4 days post-ischemic insult, albumin-immunoreactive microglia were abundant in the stratum pyramidale of the CA1 region. The present results demonstrated that albumin was newly expressed post-injury in microglia in the CA1 region, suggesting ischemia-induced neuronal loss. Albumin expression may therefore be associated with ischemia-induced delayed neuronal death in the CA1 region following transient cerebral ischemia.
\end{abstract}

\section{Introduction}

Transient cerebral ischemia leads to selective neuronal damage/death in certain brain regions, including the

Correspondence to: Professor Moo-Ho Won, Department of Neurobiology, School of Medicine, Kangwon National University, 1 Gangwondaehak-gil, Chuncheon, Gangwon 24341, Republic of Korea

E-mail:mhwon@kangwon.ac.kr

Professor Choong-Hyun Lee, Department of Pharmacy, College of Pharmacy, Dankook University, 119 Dandae, Cheonan, South Chungcheong 31116, Republic of Korea

E-mail: anaphy@dankook.ac.kr

${ }^{*}$ Contributed equally

Key words: albumin, microglia, transient cerebral ischemia, delayed neuronal death, CA1 pyramidal neurons hippocampal CA1 region $(1,2)$. In the hippocampal CA1 region, transient cerebral ischemia-induced neuronal death occurs in pyramidal neurons several days after ischemia-reperfusion, and this phenomenon is referred to as 'delayed neuronal death' (1). However, the underlying mechanisms that are associated with ischemia-induced delayed neuronal death have not yet been fully elucidated (3-5). Following the onset of transient cerebral ischemia, dysfunction of cerebral microcirculation occurs, including cerebral blood-brain barrier (BBB) breakdown and increase in BBB permeability (6-10), resulting in the extravasation of serum proteins in the perivascular space and brain parenchyma $(6,10,11)$. The dysfunction of cerebral microcirculation following ischemic insult is known to be closely associated with the ischemia-induced neuro-inflammation, cerebral edema and neuronal injury (12).

Albumin, the most abundant plasma protein, has multifunctional properties, including maintaining the osmotic pressure of plasma and specifically binding to low molecular weight molecules (13). Several studies have reported that treatment with albumin improves neurological score and decreases histopathological damage in experimental animal models of focal and global cerebral ischemia (14-17). These neuroprotective effects of albumin are thought to be due to its properties, such as its antioxidant activity, its inhibitory activity on pathological platelet aggregation, and its role in regulation of endothelial integrity $(18,19)$. However, few studies regarding albumin expression and its change in neurons or glial cells in the hippocampal CA1 region following transient cerebral ischemia exist to date. Therefore, in the present study, albumin expression in the hippocampal CA1 region of the gerbil, a popular animal model of transient cerebral ischemia (20), was examined following $5 \mathrm{~min}$ of transient cerebral ischemia. This suggests that albumin has the potential to be an attractive target for the treatment of cerebral ischemia.

\section{Materials and methods}

Experimental animals and induction of transient cerebral ischemia. Male, 6-month old Mongolian gerbils (Meriones unguiculatus) were obtained from the Experimental 
Animal Center of the Kangwon National University (Chuncheon, Republic of Korea) and were housed according to the animal handling and care guidelines as in compliance with the current international laws and policies (Guide for the Care and Use of Laboratory Animals, The National Academies Press, 8th edition, 2011) (21). The present study was approved by the Institutional Animal Care and Use Committee of Kangwon National University (Chuncheon, Korea; approval no. KW-130424-1).

The surgical procedure for transient cerebral ischemia was performed as previously described (22). In brief, the animals were anesthetized with a mixture of $2.5 \%$ isoflurane in $33 \%$ oxygen and $67 \%$ nitrous oxide. The complete interruption of blood flow mediated by the 5 min occlusion of bilateral common carotid arteries was confirmed by observing the central artery in retinae under an ophthalmoscope. Normothermic $\left(37 \pm 0.5^{\circ} \mathrm{C}\right)$ conditions were maintained prior to, during and following the surgery until the animals completely recovered from anesthesia. Sham-operated animals were subjected to the same surgical procedures except that the common carotid arteries were not occluded.

Cresyl violet $(C V)$ staining and immunohistochemistry for albumin. Sections were prepared from the sham and ischemia-operated animals ( $\mathrm{n}=7$ at each time-point) at 2 and 4 days after reperfusion, according to the previously published procedure (22). In brief, the animals were perfused transcardially with $4 \%$ paraformaldehyde in $0.1 \mathrm{M}$ phosphate-buffer $(\mathrm{pH}$ 7.4). The brain tissues were then serially sectioned into $30 \mu \mathrm{m}$ coronal sections and they were then placed into 6-well plates containing phosphate-buffered saline.

To examine cellular distribution and damage $\mathrm{CV}$ staining was performed as previously described (23). In brief, CV acetate (Sigma-Aldrich; Merck KGaA, Darmstadt, Germany) was dissolved at $1.0 \%(\mathrm{w} / \mathrm{v})$, and glacial acetic acid was added at $0.28 \%$ in this solution. The sections were stained and dehydrated by immersing in serial ethanol baths.

Albumin immunohistochemistry was carried out according to our previously published procedure (22). Briefly, sections were incubated with mouse anti-albumin (catalog no. ab19194; dilution 1:100; Abcam, Cambridge, MA, USA), exposed to biotinylated goat anti-mouse immunoglobulin (Ig) G and streptavidin peroxidase complex (Vector Laboratories, Inc., Burlingame, CA, USA) and visualized with 3,3'-diaminobenzidine. In order to confirm the cell type containing the albumin immunoreactivity, sections were also processed by double immunofluorescence staining. In brief, sections were incubated in a mixture of mouse anti-albumin (dilution 1:50; Abcam) and rabbit anti-ionized calcium-binding adapter molecule 1 (Iba-1; an established microglia marker; catalog no. 019-19741; dilution 1:100; Wako Pure Chemical Industries, Ltd., Osaka, Japan), followed by a mixture of Cy3-conjugated goat anti-mouse IgG (dilution 1:200) and fluorescein isothiocyanate-conjugated goat anti-rabbit IgG (dilution 1:200) (both from Jackson ImmunoResearch Laboratories, Inc., West Grove, PA, USA). The immunoreactions were observed under a confocal microscope (LSM 510 META NLO; Carl Zeiss AG, Oberkochen, Germany).

For the analysis of albumin immunoreactivity, six sections per animal in each group $(\mathrm{n}=7)$ were selected with $120 \mu \mathrm{m}$ intervals to quantitatively analyze albumin immunoreactivity. Digital images of the hippocampal CA1 region were captured with an AxioM1 light microscope (Carl Zeiss AG) equipped with a digital camera (Axiocam; Carl Zeiss AG). According to our previously published procedure (22), semi-quantification of the immunostaining intensities was evaluated with digital image analysis software (MetaMorph 4.01; Universal Imaging, Inc., Bedford Hills, NY, USA). The mean intensity of the albumin immunostaining in each immunoreactive structure was measured using a 0-255 gray scale system. The level of immunoreactivity was scored as follows: -, no staining (gray scale value: $\geq 200$ ) \pm , weakly positive (gray scale value: 150-199); +, moderate (gray scale value: 100-149); ++, strong (gray scale value: $50-99$ ); or +++ , very strong (gray scale value: $\leq 49$ ). The immunoreactivity scores were obtained by averaging the measurements from each animal.

\section{Results}

Delayed neuronal death. To examine ischemia-induced delayed neuronal death in the hippocampus, CV staining was performed. In the sham-operated group, $\mathrm{CV}$-positive cells were observed in all subregions of the hippocampus (Fig. 1A). In the ischemia-operated groups, the distribution pattern of $\mathrm{CV}$-positive cells was not altered at 2 days following ischemia-reperfusion compared with the sham-operated group (Fig. 1B). However, at 4 days following ischemia-reperfusion, a markedly decreased number of neurons in the stratum pyramidale of the CA1 region were stained with $\mathrm{CV}$ compared with the sham-operated group (Fig. 1C). This effect was evident only in the stratum pyramidale, as pyramidal neurons in other subregions of the hippocampus were well-stained with $\mathrm{CV}$ in the ischemia-operated and the sham-operated groups (Fig. 1C).

Albumin immunoreactivity. In the CA1 region tissue sections of the sham-operated group, albumin immunoreactivity was observed only in blood vessels, and no albumin-immunoreactive cells were observed (Fig. 2A). However, following transient cerebral ischemia, albumin immunoreactivity was generally increased in the tissue sections and observed in cells. At 2 days after ischemia-reperfusion, albumin immunoreactivity was observed in microglia-like cells in the stratum oriens and stratum radiatum of the CA1 region (Table I and Fig. 2B). At 4 days after ischemia-reperfusion, albumin immunoreactivity was further increased in microglia-like cells in the stratum oriens and stratum radiatum through the CA1 region compared with the sham and the 2 day post-ischemia groups (Table I and Fig. 2C). Notably, at 4 days after ischemia-reperfusion, strong albumin immunoreactivity was also detected in the stratum pyramidale (Table I and Fig. 2C).

To confirm the identity of the cell type that exhibited specific positive albumin immunostaining in the CA1 region tissue sections at 4 days post-ischemia, double immunofluorescence staining was performed for albumin and Iba-1, an established microglia marker. The results demonstrated that many of the albumin-immunoreactive cells were colocalized with Iba-1-positive microglia (Fig. 3), suggesting that albumin is strongly expressed by microglia in the hippocampal CA1 region following ischemic insult. 

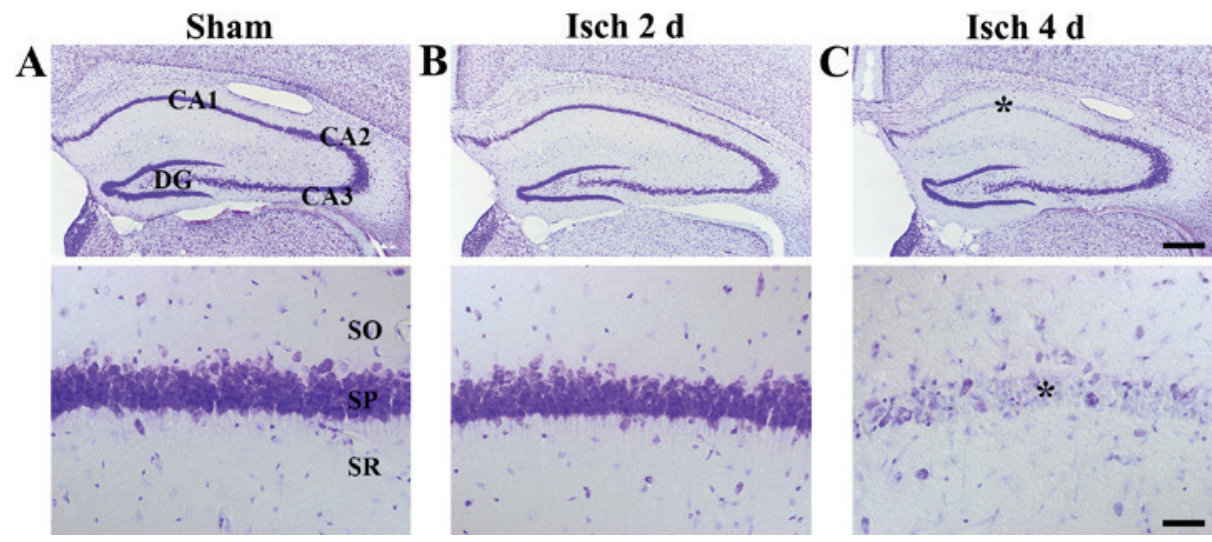

Figure 1. CV staining in the hippocampus following transient cerebral ischemia. Hippocampal tissue sections of (A) sham and ischemia-operated groups at (B) 2 days or (C) 4 days post-ischemia were stained with CV (purple signal). A significant loss of CV-positive cells is observed in the stratum pyramidale (marked by asterisk) of the CA1 region at 4 days post-ischemia. These are representative images from the analysis of 7 animals per group. Scale bar, $800 \mu \mathrm{m}$ (upper panels) and $50 \mu \mathrm{m}$ (lower panels). CV, cresyl violet; Isch $2 \mathrm{~d}$, day 2 post-ischemia; Isch $4 \mathrm{~d}$, day 4 post-ischemia; CA, conus ammonis; DG, dentate gyrus; $\mathrm{SO}$, stratum oriens; $\mathrm{SR}$, stratum radiatum.
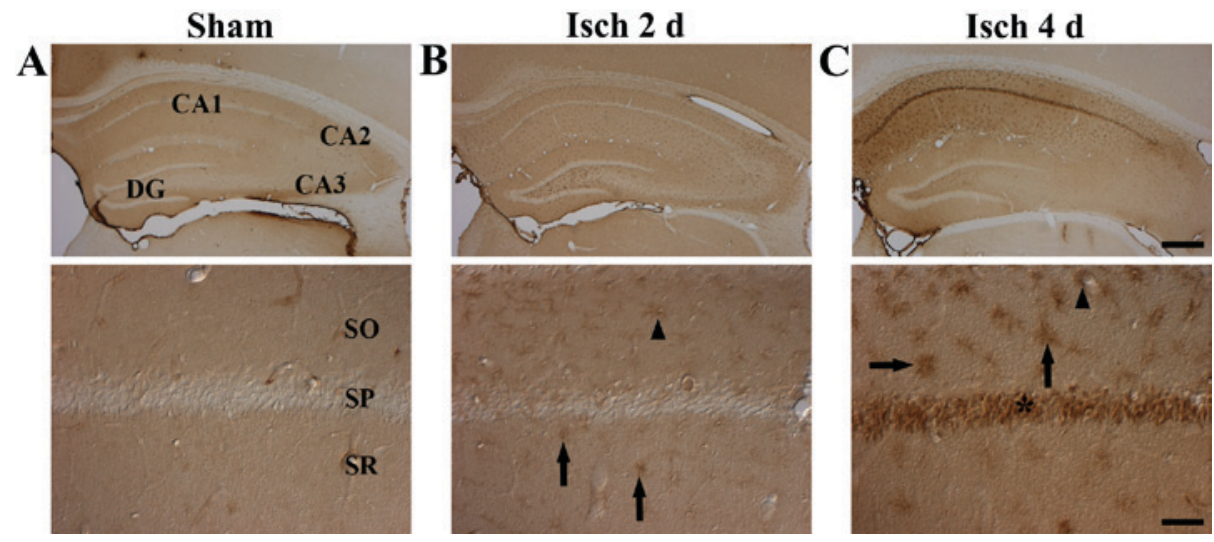

Figure 2. Albumin immunohistochemistry in the CA1 region following transient cerebral ischemia. Hippocampal tissue sections of (A) sham and ischemia-operated groups at (B) 2 days or (C) 4 days post-ischemia were immunostained for albumin protein expression (brown signal). Albumin immunoreactivity is observed in hippocampal parenchyma (arrows) as well as perivascular space (arrowheads). Asterisk indicates the albumin immunoreactivity in the stratum pyramidale. These are representative images from the analysis of 7 animals per group. Scale bar, $800 \mu \mathrm{m}$ (upper panels) and $50 \mu \mathrm{m}$ (lower panels). Isch $2 \mathrm{~d}$, day 2 post-ischemia; Isch 4 d, day 4 post-ischemia; CA, conus ammonis; DG, dentate gyrus; SO, stratum oriens; SR, stratum radiatum.

\section{Discussion}

In the present study, delayed neuronal death in the hippocampal CA1 region following transient cerebral ischemia was examined using CV staining. The results demonstrated that pyramidal neurons in the CA1 region exhibited a 'delayed neuronal death' phenotype at 4 days post-ischemic insult. This result is in agreement with the results of previously published studies $(1,2,21)$.

Maeda et al (24) demonstrated that expression of albumin is observed in the extracellular space of the brain parenchyma shortly after transient cerebral ischemia and its prompt accumulation occurred in the periphery of dendrites with neurons, likely due to the process of degeneration and death. In addition, it has been reported that BBB breakdown and extravasation of intravenously injected Evans Blue dye is observed in the forebrain 2 days after ischemic insult, and the extravasation was most severe at 4-6 days following 5 min of transient cerebral ischemia in gerbils (6). Based on these reports, the present study examined albumin immunoreactivity in the hippocampal CA1 region at 2 and 4 days after transient
Table I. Level of albumin immunoreactivity in microglia of the CA1 hippocampal region following transient cerebral ischemia.

\begin{tabular}{lccc}
\hline & \multicolumn{3}{c}{ Time after ischemia/reperfusion } \\
\cline { 2 - 4 } Region & Sham & 2 days & 4 days \\
\hline SO & - & \pm & +++ \\
SP & - & - & +++ \\
SR & - & \pm & +++ \\
\hline
\end{tabular}

Semi-quantification of the immunostaining intensity of albumin was assessed from 6 sections per animal in each group $(n=7)$. Immunoreactivity was scored as follows: negative (-), weakly positive $( \pm)$, moderate $(+)$, strong $(++)$ and very strong $(+++)$. SO, stratum oriens; SP, stratum pyramidale; SR, stratum radiatum.

cerebral ischemia. The results demonstrated that albumin immunoreactivity was first observed in Iba-1-immunoreactive 

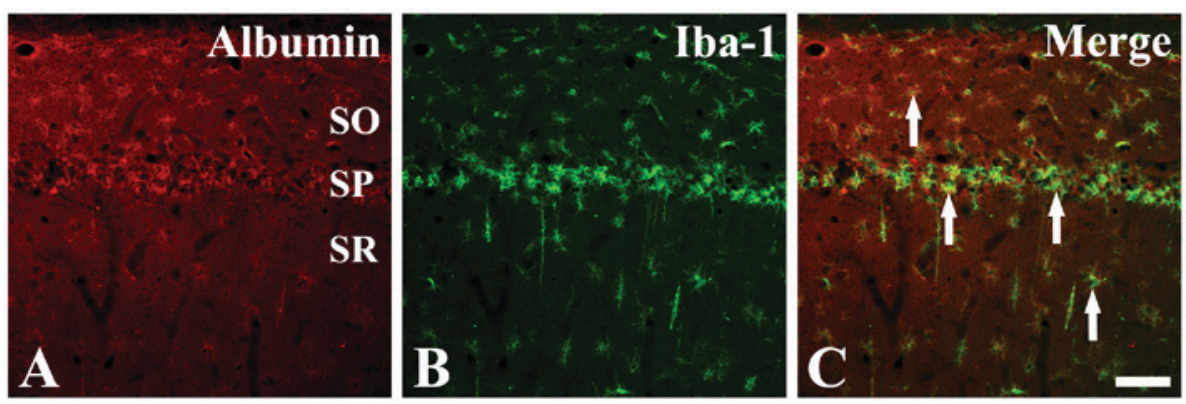

Figure 3. Albumin expression in microglia of the CA1 region following transient cerebral ischemia. Hippocampal CA1 region tissue sections from ischemia-operated group at 4 days post-ischemia were stained by immunofluorescence for (A) albumin (red) and (B) Iba-1 (microglia marker, green). (C) Albumin immunoreactivity in Iba-1-positive microglia can be observed in the merged image (arrows). These are representative images from the analysis of 7 animals per group. Scale bar, $100 \mu \mathrm{m}$. Iba-1, ionized calcium-binding adapter molecule 1; SO, stratum oriens; SP, stratum pyramidale; SR, stratum radiatum.

microglia near the microvessels in the CA1 region at 2 days after ischemia-reperfusion. In addition, albumin immunoreactivity was further increased in Iba-1-immunoreactive microglia at 4 days after ischemia-reperfusion, and at this time, strong albumin immunoreactivity was also observed in the stratum pyramidale. The present finding is in accordance with previous studies demonstrating that microglia aggregate in the stratum pyramidale of the CA1 region at 4 days after transient cerebral ischemia, the subregion where most of the pyramidal neuron death occurs $(23,25)$.

Albumin is known to induce a unique signaling cascade in microglia, which triggers microglial proliferation (26). Alonso et al (27) reported that extravasated albumin was taken up by activated microglia following ultrasound-mediated $\mathrm{BBB}$ opening. However, it has been reported that albumin is synthesized by microglia in the brain as well (28), and that its synthesis and extracellular secretion enhances microglial activation following amyloid $\beta$ protein fragment 1-42 $\left(\mathrm{A} \beta_{1-42}\right)$ or lipopolysaccharide-treatment (28). In addition, Byun et al (29) reported that albumin, synthesized mainly from activated microglia, is conjugated with advanced glycation end-product (AGE) to produce a toxic AGE-albumin, which promotes $A \beta$ polymerization and neuronal death. In the present study, ischemia-induced activated microglia were demonstrated to increase albumin immunoreactivity in the CA1 hippocampal region. The underlying mechanism for the increased albumin immunoreactivity observed in microglia only in the CA1 region following transient cerebral ischemia remains unknown. However, based on the above-mentioned literature and the our present study, it can be postulated that albumin in microglia may actively participate in the process of 'delayed neuronal death' of the CA1 pyramidal neurons following transient cerebral ischemia. By contrast, albumin is not observed in microglia in striatal parenchyma following thromboembolic middle cerebral artery occlusion in rats (30). Therefore, the expression and function of albumin in microglia may be different in various animal models of ischemia insults and should be investigated further.

In summary, the present study demonstrated that albumin immunoreactivity was newly observed in microglia in the hippocampal CA1 region following $5 \mathrm{~min}$ of transient cerebral ischemia. These results indicate that transient ischemia-induced albumin expression in microglia might be associated with ischemia-induced 'delayed neuronal death' in the hippocampal CA1 pyramidal neurons.

\section{Acknowledgements}

The present research was supported by the Bio-Synergy Research Project (grant no. NRF-2015M3A9C4076322) of the Ministry of Science, ICT and Future Planning through the National Research Foundation, and by Basic Science Research Program through the National Research Foundation of Korea funded by the Ministry of Education (grant no. NRF-2014R1A1A2058440).

\section{References}

1. Kirino T: Delayed neuronal death in the gerbil hippocampus following ischemia. Brain Res 239: 57-69, 1982.

2. Kirino T and Sano K: Selective vulnerability in the gerbil hippocampus following transient ischemia. Acta Neuropathol 62: 201-208, 1984.

3. Chan PH: Reactive oxygen radicals in signaling and damage in the ischemic brain. J Cereb Blood Flow Metab 21: 2-14, 2001.

4. Stoll G, Jander S and Schroeter M: Inflammation and glial responses in ischemic brain lesions. Prog Neurobiol 56: 149-171, 1998.

5. Won MH, Kang T, Park S, Jeon G, Kim Y, Seo JH, Choi E, Chung $M$ and Cho SS: The alterations of N-Methyl-D-aspartate receptor expressions and oxidative DNA damage in the CA1 area at the early time after ischemia-reperfusion insult. Neurosci Lett 301: 139-142, 2001 .

6. Kataoka Y, Cui Y, Yamada H, Utsunomiya K, Niiya $H$, Yanase H, Nakamura Y, Mitani A, Kataoka K and Watanabe Y: Neovascularization with blood-brain barrier breakdown in delayed neuronal death. Biochem Biophys Res Commun 273: 637-641, 2000.

7. Ritter LS, Orozco JA, Coull BM, McDonagh PF and Rosenblum WI: Leukocyte accumulation and hemodynamic changes in the cerebral microcirculation during early reperfusion after stroke. Stroke 31: 1153-1161, 2000.

8. Suchadolskiene O, Pranskunas A, Baliutyte G, Veikutis V, Dambrauskas Z, Vaitkaitis D and Borutaite V: Microcirculatory, mitochondrial, and histological changes following cerebral ischemia in swine. BMC Neurosci 15: 2, 2014.

9. Xu XS, Ma ZZ, Wang F, Hu BH, Wang CS, Liu YY, Zhao XR, An LH, Chang X, Liao FL, et al: The antioxidant Cerebralcare Granule attenuates cerebral microcirculatory disturbance during ischemia-reperfusion injury. Shock 32: 201-209, 2009.

10. Yan BY, Pan CS, Mao XW, Yang L, Liu YY, Yan L, Mu HN, Wang CS, Sun K, Liao FL, et al: Icariside II improves cerebral microcirculatory disturbance and alleviates hippocampal injury in gerbils after ischemia-reperfusion. Brain Res 1573: 63-73, 2014. 
11. He Z, He B, Behrle BL, Fejleh MP, Cui L, Paule MG and Greenfield LJ: Ischemia-induced increase in microvascular phosphodiesterase 4D expression in rat hippocampus associated with blood brain barrier permeability: Effect of age. ACS Chem Neurosci 3: 428-432, 2012.

12. Rosenberg GA and Yang Y: Vasogenic edema due to tight junction disruption by matrix metalloproteinases in cerebral ischemia. Neurosurg Focus 22: E4, 2007.

13. Petricoin EF, Belluco C, Araujo RP and Liotta LA: The blood peptidome: A higher dimension of information content for cancer biomarker discovery. Nat Rev Cancer 6: 961-967, 2006.

14. Belayev L, Liu Y, Zhao W, Busto R and Ginsberg MD: Human albumin therapy of acute ischemic stroke: Marked neuroprotective efficacy at moderate doses and with a broad therapeutic window. Stroke 32: 553-560, 2001.

15. Belayev L, Saul I, Huh PW, Finotti N, Zhao W, Busto R and Ginsberg MD: Neuroprotective effect of high-dose albumin therapy against global ischemic brain injury in rats. Brain Res 845: 107-111, 1999.

16. Eady TN, Khoutorova L, Obenaus A, Mohd-Yusof A, Bazan NG and Belayev L: Docosahexaenoic acid complexed to albumin provides neuroprotection after experimental stroke in aged rats. Neurobiol Dis 62: 1-7, 2014.

17. Yao X, Miao W, Li M, Wang M, Ma J, Wang Y, Miao L and Feng H: Protective effect of albumin on VEGF and brain edema in acute ischemia in rats. Neurosci Lett 472: 179-183, 2010.

18. Belayev L, Pinard E, Nallet H, Seylaz J, Liu Y, Riyamongkol P, Zhao W, Busto R and Ginsberg MD: Albumin therapy of transient focal cerebral ischemia: In vivo analysis of dynamic microvascular responses. Stroke 33: 1077-1084, 2002.

19. Belayev L, Zhao W, Pattany PM, Weaver RG, Huh PW, Lin B, Busto $\mathrm{R}$ and Ginsberg MD: Diffusion-weighted magnetic resonance imaging confirms marked neuroprotective efficacy of albumin therapy in focal cerebral ischemia. Stroke 29: 2587-2599, 1998

20. Strosznajder RP, Gadamski R, Czapski GA, Jesko H and Strosznajder JB: Poly(ADP-ribose) polymerase during reperfusion after transient forebrain ischemia: Its role in brain edema and cell death. J Mol Neurosci 20: 61-72, 2003.

21. Institute of Laboratory Animal Research, Committee for the Update of the Guide for the Care and Use of Laboratory Animals, National Research Council: Guide for the Care and Use of Laboratory Animals. 8th edition. National Academies Press, Washington, DC, pp220, 2011.
22. Lee CH, Park JH, Cho JH, Ahn JH, Yan BC, Lee JC, Shin MC, Cheon SH, Cho YS, Cho JH, et al: Changes and expressions of Redd1 in neurons and glial cells in the gerbil hippocampus proper following transient global cerebral ischemia. J Neurol Sci 344: 43-50, 2014.

23. Park JH, Shin BN, Chen BH, Kim IH, Ahn JH, Cho JH, Tae HJ, Lee JC, Lee CH, Kim YM, et al: Neuroprotection and reduced gliosis by atomoxetine pretreatment in a gerbil model of transient cerebral ischemia. J Neurol Sci 359: 373-380, 2015.

24. Maeda M, Akai F, Nishida S and Yanagihara T: Intracerebral distribution of albumin after transient cerebral ischemia: Light and electron microscopic immunocytochemical investigation. Acta Neuropathol 84: 59-66, 1992.

25. Lee CH, Park JH, Yoo KY, Choi JH, Hwang IK, Ryu PD, Kim DH, Kwon YG, Kim YM and Won MH: Pre- and post-treatments with escitalopram protect against experimental ischemic neuronal damage via regulation of BDNF expression and oxidative stress. Exp Neurol 229: 450-459, 2011.

26. Hooper C, Taylor DL and Pocock JM: Pure albumin is a potent trigger of calcium signalling and proliferation in microglia but not macrophages or astrocytes. J Neurochem 92: 1363-1376, 2005.

27. Alonso A, Reinz E, Fatar M, Hennerici MG and Meairs S: Clearance of albumin following ultrasound-induced blood-brain barrier opening is mediated by glial but not neuronal cells. Brain Res 1411: 9-16, 2011.

28. Ahn SM, Byun K, Cho K, Kim JY, Yoo JS, Kim D, Paek SH, Kim SU, Simpson RJ and Lee B: Human microglial cells synthesize albumin in brain. PLoS One 3: e2829, 2008

29. Byun K, Bayarsaikhan E, Kim D, Kim CY, Mook-Jung I, Paek SH, Kim SU, Yamamoto T, Won MH, Song BJ, et al: Induction of neuronal death by microglial AGE-albumin: Implications for Alzheimer's disease. PLoS One 7: e37917, 2012.

30. Lehmann J, Härtig W, Seidel A, Füldner C, Hobohm C, Grosche J, Krueger $\mathrm{M}$ and Michalski D: Inflammatory cell recruitment after experimental thromboembolic stroke in rats. Neuroscience 279: 139-154, 2014. 